\title{
Theoretical Conformational Analysis on Gramicidin A Model Polypeptide with L-Ala-D-Ala Repeated Sequence
}

\author{
Masahito OKA, ${ }^{\dagger}$ Yoshihiro BABA, Akihiro Kagemoto, \\ and Akio NAKAJIMA* \\ Departments of General Education and \\ * Applied Chemistry, Osaka Institute of Technology, \\ Omiya, Asahi-ku, Osaka 535, Japan
}

(Received July 10, 1989)

\begin{abstract}
Theoretical conformational analysis was carried out for Ac-(L-Ala-D-Ala) 12- $^{-}$ NHMe, a model polypeptide of gramicidin A, using ECEPP and the conformational energy minimization procedure. Through the analysis, the hypothesis on the interaction in polypeptide molecules was used. Calculated results showed that right-handed $\beta^{4.6}$-helical structure is the most stable conformation, and that $\beta^{6.2}$-helical and $\alpha$-helical structures are also stable conformations.

KEY WORDS Conformational Analysis / ECEPP / Poly(L-Ala-D-Ala) /

Gramicidin A / Helical Structure / $\beta$-Helix /
\end{abstract}

Gramicidin A is a linear pentadecapeptide with the alternating $\mathrm{L}-$ and $\mathrm{D}$-hydrophobic amino acid sequence, L-Val-Gly-L-Ala-D-LeuL-Ala-D-Val-L-Val-D-Val-L-Trp-D-Leu-L-TrpD-Leu-L-Trp-D-Leu-L-Trp, and also forms transmembrane channels which select transport cations across the lipid bilayer. ${ }^{1-3}$ Four helical models of gramicidin A were proposed, and two of them have been experimentally observed. That is, the head-to-head $\beta^{6}$-helical dimer at the amino termini ${ }^{4}$ was detected by conductance study, $\mathrm{CD}$ and ${ }^{1} \mathrm{H}$ NMR measurements in organic solvents ${ }^{4}$ and ${ }^{13} \mathrm{C}$ and ${ }^{19}$ F NMR measurements in phosphatidylcholine vesicles. ${ }^{6}$ The antiparallel doublestranded $\beta$-helix ${ }^{7}$ was also detected by IR and CD measurements in alcohol and dioxane ${ }^{j}$ and X-ray diffraction measurements in crystal state..$^{8,9}$

Copolypeptides and cooligopeptides composed of alternating $\mathrm{L}$ - and D-amino acids were synthesized and their conformations in so- lutions and solid states were experimentally investigated, and several kinds of single $\mathrm{e}^{10-16}$ and double ${ }^{13-21}$ stranded conformations were experimentally proposed.

Helical conformations of polypeptide chains with alternating $\mathrm{L}^{-}$and $\mathrm{D}$-residues were theoretically analyzed, ${ }^{22-25}$ and several kinds of single- and double-stranded helical conformations were investigated. Ramachandran and Chandrasekaran ${ }^{22}$ theoretically proposed the LD-ribbon structures and $\mathrm{LD}_{3}\left(\beta^{4}\right), \mathrm{LD}_{4}\left(\beta^{6}\right)$, and $\mathrm{LD}_{5}\left(\beta^{8}\right)$-helical structures based on analysis of the possible backbone/backbone hydrogen-bonds of poly(L-Ala-D-Ala). Through grid analysis, it was found that the most stable conformation among them is $\mathrm{LD}_{3}$-helix ( $\beta^{4}$-helix). Hesselink and Scheraga ${ }^{23}$ theoretically analyzed six helical conformations (i.e., $\alpha$-helix, LD-ribbon, and LD-helix ( $\beta^{6}$-helix) and their enantiomers) of (L-Ala-D-Ala) ${ }_{6}-\mathrm{L}-$ Ala with conformational energy minimization procedure. They showed that $\alpha$-helix is

\footnotetext{
† To whom all correspondence should be addressed.
} 
the most stable conformation among six helical conformations and also indicated that poly(L-Ala-D-Ala) can adopt an $\alpha$-helical conformation. De Santis et al. ${ }^{24}$ investigated the helical conformations of the regular enantiomeric sequence using the eigenvalue-eigenvectors solutions of the Eyring-transformation matrix, and proposed the $\mathrm{R}_{\beta} 4.8, \mathrm{R}_{\beta} 6.2$, $\mathrm{R}_{\beta} 8.2, \mathrm{~L}_{\beta} 4.8, \mathrm{~L}_{\beta} 6.2$ and $\mathrm{L}_{\beta} 8$.2-helices (their notation $\mathrm{R}_{\beta} \mathrm{x}$ and $\mathrm{L}_{\beta} \mathrm{x}$ correspond to rightand left-handed $\beta^{\mathrm{x}}$-helices, respectively), and $\beta-\mathrm{R}_{\alpha}, \beta-\mathrm{L}_{\alpha}$ and $\mathrm{R}_{\alpha}-\mathrm{L}_{\alpha}$ structures. ColonnaCesari et al. ${ }^{25}$ calculated the conformational energy of the single- and double-stranded helical conformations of (L-Ala-D-Ala $)_{8}$ with conformational energy minimization procedure. They obtained the $\alpha$-helix as the most stable single-stranded conformation among $\alpha$-helix, $\pi_{\mathrm{DL}}{ }^{4.4}$-helix $\left(\beta^{4.4}\right.$-helix $)$ and $\pi_{\mathrm{DL}}{ }^{6.3}$-he$\operatorname{lix}\left(\beta^{6.3}\right.$-helix $)$. Venkatachalam and $\mathrm{Urry}^{26}$ calculated the conformational energies of leftand right-handed $\beta^{6}$-helices of pentadecapeptide having the sequence corresponding to gramicidin A with 384 side-chain conformational combinations, and showed that the left-handed head-to-head dimer is more stable structure than the right-handed one.

Theoretical conformational analysis of AcL-Ala-D-Ala-NHMe was carried out by Oka and Nakajima, ${ }^{27}$ to show that L-Ala-D-Ala sequence indicates a trend forming bend conformations, and type II $\beta$-bend conformation is the most stable conformation among them. Calculated results ${ }^{27}$ also showed that Ac-LAla-D-Ala-NHMe has many stable conformations as shown in Table II of reference 27 (37 minimum conformations with $\Delta E<$ $3 \mathrm{kcal} \mathrm{mol}^{-1}$ ) and there are no specific conformations having dominantly high statistical weight with intra-sequence interactions in L-Ala-D-Ala sequence (ex., statistical weight of the lowest-energy conformation is 0.192 ).

To obtain basic information on the native structure of gramicidin A which is stablilized by intra- and intermolecular interactions, single-stranded helical conformations of
poly(L-Ala-D-Ala) were theoretically analyzed by the conformational energy minimization procedure taking into consideration all intramolecular interactions in this paper. This analysis is based on the hypothesis which has been shown for peptide, polypeptide and protein systems. ${ }^{28}$

\section{THEORETICAL}

All conformational energy calculations were carried out with ECEPP. ${ }^{29}$ Minimization was carried out with the Powell algorithm ${ }^{30}$ until conformational energy did not change by more than $0.001 \mathrm{kcal} \mathrm{mol}^{-1}$ between successive iterations. During minimizations, all $\phi, \psi$, and $\chi^{1}$ for L-Ala and D-Ala residues were allowed to vary. All other backbone dihedral angles were fixed at $180^{\circ}$.

Two sets of starting conformations were used. The first set was obtained by the following method. All minimum energy conformations of Ac-L-Ala-D-Ala-NHMe $(\Delta E<$ $\left.5 \mathrm{kcal} \mathrm{mol}^{-1}\right)^{27}$ were used as the starting conformations of the peptide having two repeating units of L-Ala-D-Ala, i.e., Ac- $(\mathrm{L}-$ Ala-D-Ala $)_{2}$-NHMe. During minimization, the corresponding dihedral angles of each repeating unit (for example, $\phi_{\mathrm{L}-\mathrm{Ala}}$ in the first repeating unit and $\phi_{\mathrm{L}-\mathrm{Ala}}$ in the second unit) were treated as a common variable of minimization; that is, the condition of helical conformation, was used. Then, all minimum-energy conformations of Ac-(L-Ala-DAla $)_{2}$-NHMe $\left(\Delta E<10 \mathrm{kcal} \mathrm{mol}^{-1}\right)$ were used as starting conformations for the minimization of conformational energy of Ac-(L-Ala-DAla) $)_{12}$-NHMe.

Selection of the starting conformations in the first set is based on the hypothesis shown for the peptide, polypeptide and protein systems. ${ }^{28}$ That is, (1) the primary ensemble of the stable conformations of polypeptides is primarily decided by the short-range interactions, and (2) relative stabilities of these conformations are changed, and the particular con- 
formations are stabilized by medium- and long-range interactions. Through this method (the three-steps method), missing the stable conformations by trapping the local minimum caused by the tremendous atomic overlaps at the primary stage of minimization could be avoided and minimization could be effectively carried out by saving computation time.

The second set was selected by the following method. Conformational energy of Ac(L-Ala-D-Ala $)_{12}$-NHMe was calculated by changing $\phi_{\mathrm{L}-A l a}$ and $\psi_{\mathrm{L}-\mathrm{Ala}}$ at $15^{\circ}$ intervals and fixing $\left(\phi_{\text {D-Ala }}, \psi_{\text {D-Ala }}\right)$ to the energy minima of Ac-D-Ala-NHMe (i.e., $\left(84^{\circ},-80^{\circ}\right)$, $\left(-54^{\circ},-57^{\circ}\right),\left(151^{\circ},-73^{\circ}\right),\left(74^{\circ}, 45^{\circ}\right),\left(154^{\circ}\right.$, $\left.-153^{\circ}\right),\left(158^{\circ}, 58^{\circ}\right),\left(-64^{\circ},-178^{\circ}\right)$, and $\left(-78^{\circ}, 64^{\circ}\right)$ ), and fixing $\chi_{\text {LAla }}^{1}$ and $\chi^{1}$ D-Ala $=60^{\circ}$ and all other dihedral angles to $180^{\circ}$. 54 local minimum conformations in $\left(\phi_{\mathrm{L} \text {-Ala }}\right.$, $\left.\psi_{\text {L-Ala }}\right)$ space were selected as starting conformations.

Energy minimizations were carried out for all conformations in the above two startingconformaion sets with the condition of a helical conformation.
A bend (occurring at $i+1$ and $i+2$ th residues) is defined as a conformation in which $R \leqq 7 \AA$ ( $R$ is the distance between $i$ th $C^{\alpha}$ and $i+3$ th $\mathrm{C}^{\alpha}$ atoms.) and also classified into eleven types given in Table I of ref 31. A polar hydrogen atom and an oxygen or nitrogen atom with an interatomic distance of less than $2.3 \AA$ are regarded to be hydrogen-bonded. Conformational space is devided into 16 regions with the conformational letter codes shown in Figure 1 of ref 32. The conformational energy per whole molecule, $\Delta E$, is defined by $\Delta E=E-E_{\mathrm{o}}$, where $E_{\mathrm{o}}$ is the value of $E$ at the global minimum on the potential energy surface of the particular molecules, and $\Delta E_{\mathrm{res}}$ is defined by $\Delta E_{\mathrm{res}}=\Delta E / m$, where $m$ is number of residues of the molecule. $\beta^{x}$-helix is defined as a helix which has a spiral structure with $x$ residues per turn.

\section{RESULTS}

Stable Conformations of Ac-(L-Ala-D-Ala $)_{2}-$ NHMe

A total of 35 energy minima of Ac-(L-Ala-

Table I. Calculated minimum energy conformations ${ }^{\mathrm{a}}$ of Ac-(L-Ala-D-Ala) $)_{12}-\mathrm{NHMe}$

\begin{tabular}{|c|c|c|c|c|c|c|c|c|}
\hline $\begin{array}{l}\text { Conformational } \\
\text { letter code }\end{array}$ & $\frac{\Delta E_{\mathrm{res}}^{\mathrm{b}}}{\mathrm{kcal} \mathrm{mol}^{-1}}$ & $\begin{array}{l}\text { Helix }^{c} \\
\text { type }\end{array}$ & $n^{\mathrm{d}}$ & $h^{\mathrm{e}}$ & $\phi_{\mathrm{L}-\mathrm{Ala}}$ & $\psi_{\text {L-Ala }}$ & $\phi_{\mathrm{D}-\mathrm{Ala}}$ & $\psi_{\text {D-Ala }}$ \\
\hline$C D^{*}$ & 0.00 & $\beta^{4.6} \quad(\mathrm{R})$ & 4.57 & 1.24 & -83 & 72 & 153 & -75 \\
\hline CE* & 0.19 & $\beta^{6.2}(\mathrm{~L})$ & 6.22 & 0.74 & -97 & 76 & 159 & -168 \\
\hline$A^{*} A^{*}$ & 0.23 & $\alpha \quad(\mathrm{L})$ & 3.59 & 1.48 & 54 & 51 & 66 & 37 \\
\hline AA & 0.24 & $\alpha \quad$ (R) & 3.59 & 1.48 & -66 & -37 & -54 & -51 \\
\hline CE* & 0.66 & $\beta^{5.5} \quad(\mathrm{~L})$ & 5.55 & 0.89 & -88 & 65 & 147 & -163 \\
\hline $\mathrm{EE}^{*}$ & 1.09 & $\beta^{8.2}(\mathrm{~L})$ & 8.21 & 0.64 & -148 & 119 & 155 & -144 \\
\hline $\mathrm{EE}^{*}$ & 1.14 & $\beta^{10.2}(\mathrm{~L})$ & 10.20 & 0.52 & -154 & 138 & 154 & -151 \\
\hline $\mathrm{EE}^{*}$ & 1.25 & $\beta^{12.2}(\mathrm{~L})$ & 12.17 & 0.43 & -157 & 149 & 155 & -156 \\
\hline AD* & 1.87 & $\beta^{5.0} \quad(\mathrm{~L})$ & 4.98 & 1.49 & -76 & -35 & 156 & -89 \\
\hline $\mathrm{CA}^{*}$ & 1.93 & $\beta^{4.0}(\mathrm{~L})$ & 4.01 & 2.17 & -68 & 105 & 77 & 48 \\
\hline$C D^{*}$ & 2.07 & $\beta^{4.3}(\mathrm{R})$ & 4.30 & 2.15 & -71 & 96 & 150 & -39 \\
\hline
\end{tabular}

a All minima with $\Delta E_{\text {res }}<2.5 \mathrm{kcal} \mathrm{mol}^{-1}$ are shown, but only the lowest-energy minimum in each helix-group constituted by the helices with common number of residues per turn is presented. For $\alpha$-helices, right- and lefthanded helices are shown.

b $E_{0}=24.07 \mathrm{kcal} \mathrm{mol}^{-1} . \Delta E_{\mathrm{res}}=\left(E-E_{0}\right) / 24$.

c Helix sence is abbreviated as $\mathrm{R}$ or $\mathrm{L}$ for right- or left-hand, respectively.

d Number of residues per turn.

e Rise per residue. 
D-Ala $)_{2}-$ NHMe was found in $\left.\Delta E<10 \mathrm{kcal} \phi_{\mathrm{D}-\mathrm{Ala}}, \psi_{\mathrm{D}-\mathrm{Ala}}\right)=\left(-96^{\circ}, 72^{\circ}, 75^{\circ},-103^{\circ}\right)$. This $\mathrm{mol}^{-1}$. The lowest-energy conformation is conformation was obtained from the second the $\mathrm{CC}^{*}$ conformation with $\left(\phi_{\text {LAla }}, \psi_{\text {L-Ala }}\right.$, and fifth low-energy conformations of Ac-



(a)

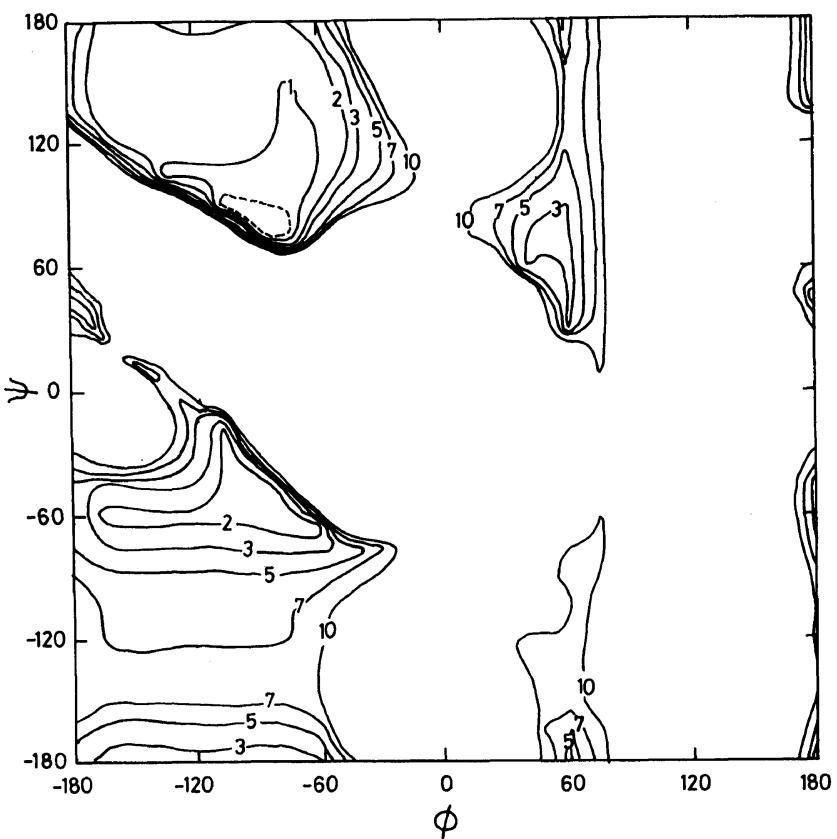

(b) 


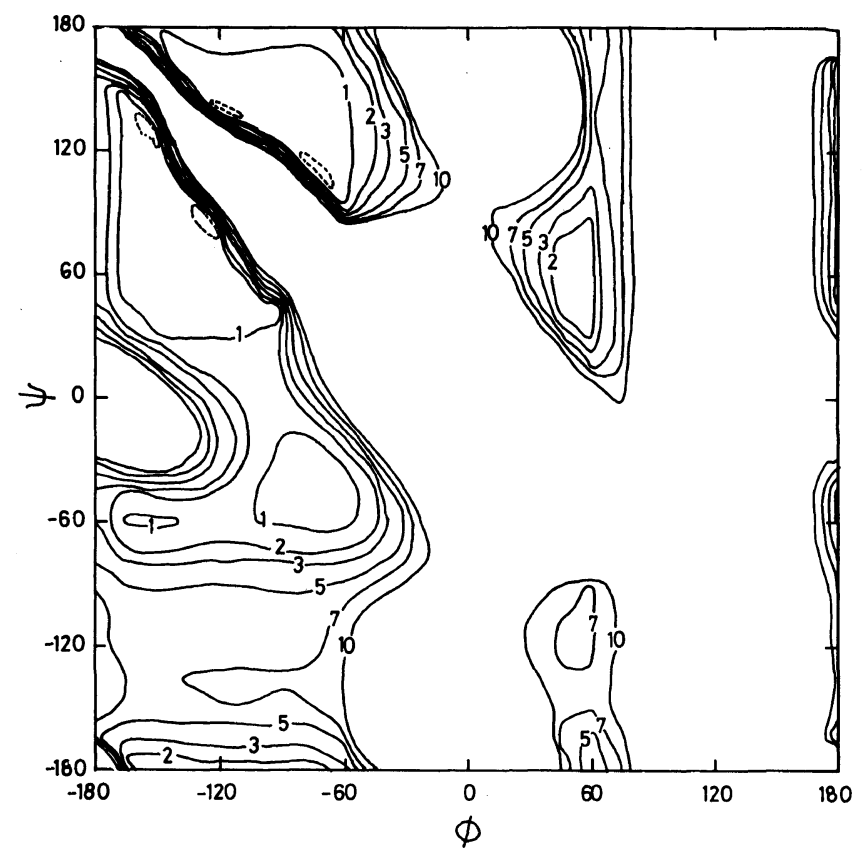

(c)

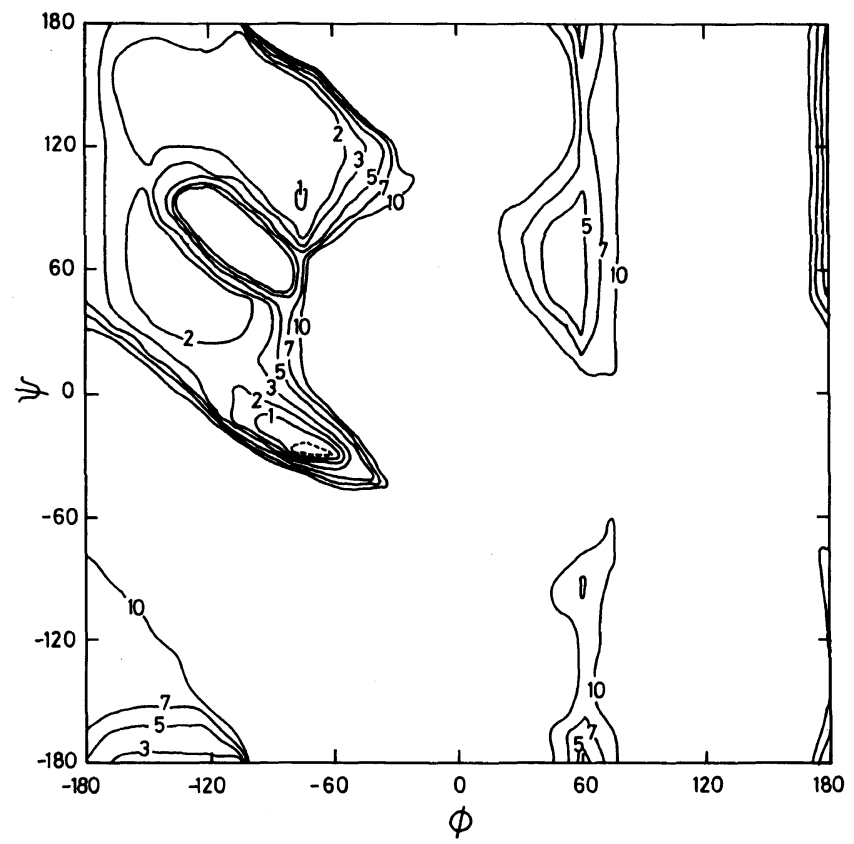

(d)

Figure 1. Energy contour $(\phi, \psi)$ maps of L-Ala residue of Ac-(L-Ala-D-Ala) $)_{12}-\mathrm{NHMe}$ at $15^{\circ}$ intervals for the specified $(\phi, \psi)$ of $\mathrm{D}$-Ala residue with the condition of helical conformation and fixing all $\chi^{1}=60^{\circ}$. $E_{\min }$ is the minimum energy in each map. (a): $\left(\phi_{\mathrm{D}-\mathrm{Ala}}, \psi_{\mathrm{D}-\mathrm{Ala}}\right)=\left(84^{\circ},-80^{\circ}\right), E_{\min }=40.86 \mathrm{kcal} \mathrm{mol}^{-1}$. (b): $\left(\phi_{\mathrm{D}-\mathrm{Ala}}, \quad \psi_{\mathrm{D}-\mathrm{Ala}}\right)=\left(151^{\circ},-73^{\circ}\right), E_{\min }=45.41 \mathrm{kcal} \mathrm{mol}^{-1} .(\mathrm{c}):\left(\phi_{\mathrm{D}-\mathrm{Ala}}, \quad \psi_{\mathrm{D}-\mathrm{Ala}}\right)=\left(154^{\circ},-153^{\circ}\right), E_{\min }=$ $60.49 \mathrm{kcal} \mathrm{mol}^{-1}$. (d): $\left(\phi_{\mathrm{D}-\mathrm{Ala}}, \psi_{\mathrm{D}-\mathrm{Ala}}\right)=\left(-54^{\circ},-57^{\circ}\right), E_{\min }=40.91 \mathrm{kcal} \mathrm{mol}^{-1}$. 
L-Ala-D-Ala-NHMe, that is $\mathrm{CC}^{*}\left(-83^{\circ}, 79^{\circ}\right.$, $\left.84^{\circ},-78^{\circ}\right)$ and $\mathrm{CF}^{*}\left(-84^{\circ}, 76^{\circ}, 74^{\circ}\right.$, $\left.-143^{\circ}\right)$, respectively. The second low-energy conformation is $\mathrm{CD}^{*}\left(-84^{\circ}, 73^{\circ}, 147^{\circ}\right.$, $-71^{\circ}$ ) obtained from the third conformation of Ac-L-Ala-D-Ala-NHMe with $\mathrm{CD}^{*}\left(-80^{\circ}\right.$, $\left.83^{\circ}, 153^{\circ},-49^{\circ}\right)$. The most stable conformation of Ac-L-Ala-D-Ala-NHMe, CA* $\left(-71^{\circ}\right.$, $\left.99^{\circ}, 77^{\circ}, 36^{\circ}\right)$ is slightly destabilized to the 4 th conformation of Ac-(L-Ala-D-Ala) $)_{2}$ $\mathrm{NHMe}, \mathrm{CA}^{*}\left(-73^{\circ}, 98^{\circ}, 77^{\circ}, 42^{\circ}\right)$ with $\Delta E=1.89 \mathrm{kcal} \mathrm{mol}^{-1}$. These results indicate that the low-energy conformations stabilized by the short-range interactions change their conformations by additional further-range interactions and also change their relative stabilities.

Local Minima in $\left(\phi_{\mathrm{L}-\mathrm{Ala}}, \psi_{\mathrm{L}-\mathrm{Ala}}\right)$ Maps of Ac(L-Ala-D-Ala $)_{12}$-NHMe for the Specified Backbone Conformation of D-Ala Residue

Four typical $(\phi, \psi)$ maps of the L-Ala residue of Ac-(L-Ala-D-Ala $)_{12}-\mathrm{NHMe}$ with the specified values of $\left(\phi_{\text {D-Ala }}, \psi_{\text {D-Ala }}\right)=\left(84^{\circ}\right.$, $\left.-80^{\circ}\right),\left(151^{\circ},-73^{\circ}\right),\left(154^{\circ},-153^{\circ}\right)$, and $\left(-54^{\circ},-57^{\circ}\right)$ under the condition of helical conformation are shown in Figures $1 \mathrm{a}, 1 \mathrm{~b}, 1 \mathrm{c}$, and $1 \mathrm{~d}$. The global minimum is found in the $\mathrm{D}$ region of Figure 1a, i.e., $\left(\phi_{\mathrm{L}-\mathrm{Ala}}, \psi_{\mathrm{L}-\mathrm{Ala}}, \phi_{\mathrm{D}-\mathrm{Ala}}\right.$, $\left.\psi_{\text {D-Ala }}\right)=\left(-150^{\circ}, 75^{\circ}, 84^{\circ},-80^{\circ}\right)$, and its conformational enrgy is $40.86 \mathrm{kcal} \mathrm{mol}^{-1}$. This global minimum conformation forms a lefthanded $\beta^{4.5}$-helix. Figure 1 indicates that the low-energy regions of L-Ala residues depend on the conformation of D-Ala residues, and that they are fairly different from the lowenergy regions in $(\phi, \psi)$ space of poly( $\mathrm{L}-$ Ala). ${ }^{33,34}$ Figures $1 \mathrm{a}, 1 \mathrm{~b}$, and $1 \mathrm{c}$ also indicate that a low-energy trough exists in the $\mathrm{CE}^{*}$, $\mathrm{DE}^{*}, \mathrm{EE}^{*}, \mathrm{EC}^{*}$, and $\mathrm{DE}^{*}$ regions along the edge of high-energy regions in $\left(\phi_{\mathrm{L}-\mathrm{Ala}}, \psi_{\mathrm{L}-\mathrm{Ala}}\right.$, $\left.\phi_{\text {D-Ala, }} \psi_{\text {D-Ala }}\right)$ conformational space. As already mentioned in the THEORETICAL section, 54 local minima found in the $\left(\phi_{\mathrm{L}-\mathrm{Ala}}, \psi_{\mathrm{L}-\mathrm{Ala}}\right.$, $\left.\phi_{\text {D-Ala }}, \psi_{\text {D-Ala }}\right)$ space were the starting conformations of energy minimization of Ac-(L-Ala-
D-Ala) $)_{12}$-NHMe.

Stable Conformations of Ac-(L-Ala-D-Ala $)_{12^{-}}$ NHMe

In Table I, all stable conformations of Ac(L-Ala-D-Ala $)_{12}-\mathrm{NHMe}$ with $\Delta E_{\mathrm{res}}<2.5 \mathrm{kcal}$ $\mathrm{mol}^{-1}$ are shown, but only the lowest-energy conformation in each helix-group constituted by the helices with common number of residues per turn is presented with one exception that both of right- and left-handed $\alpha$-helix are shown.

The lowest-energy conformation is $\mathrm{CD}^{*}$ with $\left(\phi_{\text {L-Ala }}, \psi_{\text {L-Ala }}, \phi_{\text {D-Ala }}, \psi_{\text {D-Ala }}\right)=\left(-83^{\circ}, 72^{\circ}\right.$, $\left.153^{\circ},-75^{\circ}\right)$, and its enantiomer is $\mathrm{DC}^{*}$ with $\left(-152^{\circ}, 75^{\circ}, 83^{\circ},-72^{\circ}\right)$ and their energy differences $\Delta E_{\text {res }}=0.07 \mathrm{kcal} \mathrm{mol}^{-1}$. Both of them are $\beta^{4.6}$-helices composed of consecutive bend conformations, i.e., type IV bend at L-Ala-D-Ala and D-Ala-L-Ala dipeptide sequences. The $\mathrm{CD}^{*}$ conformation is the righthanded helix shown in Figure 2a (All molecular diagrams in this paper are described by PEPMOD program. ${ }^{35}$ ), and its enantiomer $D * C$ conformation is a left-handed helix. The CD* conformation is the third lowenergy conformation $\left(\Delta E=0.34 \mathrm{kcal} \mathrm{mol}^{-1}\right)$ of Ac-L-Ala-D-Ala-NHMe ${ }^{27}$ and the second lowenergy conformation $\left(\Delta E_{\mathrm{res}}=0.02 \mathrm{kcal} \mathrm{mol}^{-1}\right)$ of Ac-(L-Ala-D-Ala) $)_{2}$-NHMe. Small change of dihedral angles of L-Ala and D-Ala residues was found between Ac-L-Ala-D-Ala-NHMe and Ac-(L-Ala-D-Ala $)_{12}$-NHMe, i.e., $\left(\Delta \phi_{\mathrm{L}-\mathrm{Ala}}\right.$, $\left.\Delta \psi_{\text {L-Ala }}, \Delta \phi_{\text {D-Ala }}, \Delta \psi_{\text {D-Ala }}\right)=\left(-3^{\circ},-11^{\circ}, 0^{\circ}\right.$, $\left.-26^{\circ}\right)$. The $\mathrm{CD}^{*}$ conformation is stabilized by two kinds of hydrogen bonds, $\left(\mathrm{L}-\mathrm{Ala}_{i+5}\right) \mathrm{NH} \cdots \mathrm{OC}\left(\mathrm{D}-\mathrm{Ala}_{i}\right)$ and $\left(\mathrm{D}-\mathrm{Ala}_{i+2}\right)$ $\mathrm{NH} \cdots \mathrm{OC}\left(\mathrm{D}-\mathrm{Ala} \mathrm{a}_{i}\right)$. The DC* conformation is the 8th low-energy conformation $(\Delta E=$ $1.00 \mathrm{kcal} \mathrm{mol}^{-1}$ ) of Ac-L-Ala-D-Ala-NHMe ${ }^{27}$, and no $\mathrm{DC}^{*}$ conformations were found for Ac-(L-Ala-D-Ala) ${ }_{2}$ NHMe. The 5 th conformation $\left(\mathrm{CF}^{*}\right)$ of dipeptide shifts to the $\mathrm{CC}^{*}$ conformation (the lowest-energy conformation of Ac-(L-Ala-D-Ala) $\left.{ }_{2}-\mathrm{NHMe}\right)$ by interunit interactions with change in their dihedral 

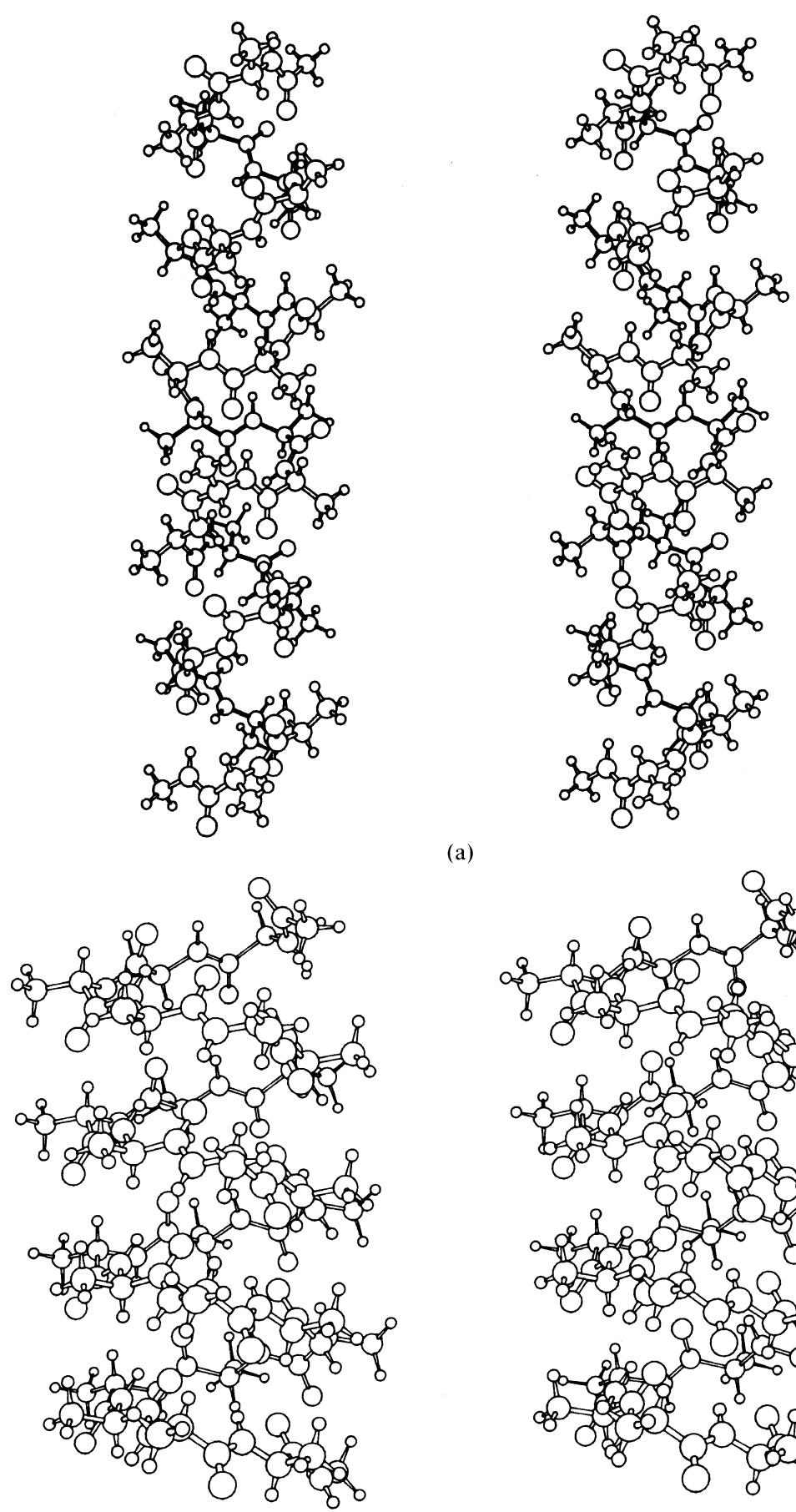

(a)

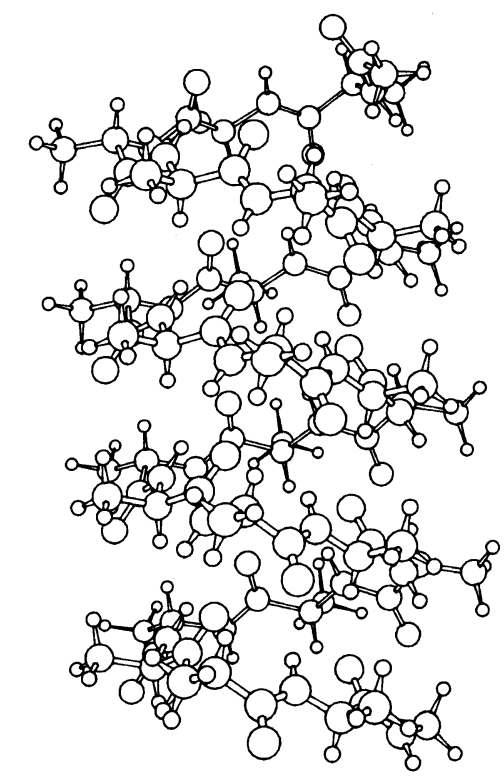

(b)

Figure 2. Helical conformations found in relatively low-energy regions. (a): Right-handed $\beta^{4.6}$-helical conformation with lowest-energy. The $\phi$ and $\psi$ of L-Ala and D-Ala residues are $\left(-83^{\circ}, 72^{\circ}, 153^{\circ},-75^{\circ}\right)$ and the corresponding conformational letter code is $\mathrm{CD}^{*}$. (b): Left-handed $\beta^{6.2}$-helical conformation with $\Delta E_{\mathrm{res}}=0.19 \mathrm{kcal} \mathrm{mol}^{-1},\left(-97^{\circ}, 76^{\circ}, 159^{\circ},-168^{\circ}\right)$ and $\mathrm{CE}^{*}$. 
angles $\left(\Delta \phi_{\text {L-Ala }}, \Delta \psi_{\text {L-Ala }}, \Delta \phi_{\text {D-Ala }}, \Delta \psi_{\text {D-Ala }}\right)=$ $\left(-12^{\circ},-4^{\circ}, 1^{\circ}, 40^{\circ}\right)$. With further-range inter-unit interactions in Ac-(L-Ala-D-Ala) ${ }_{12}-$ $\mathrm{NHMe}$, the $\mathrm{CC}^{*}$ conformation shifts to the $\mathrm{DC}^{*}$ conformation with change of their dihedral angles $\left(-56^{\circ}, 3^{\circ}, 8^{\circ}, 31^{\circ}\right)$. The DC* conformation is stabilized by two kinds of hydrogen bonds (D-Ala $\left.{ }_{i+5}\right) \mathrm{NH} \cdots \mathrm{OC}$ $\left(\mathrm{L}-\mathrm{Ala}_{i}\right)$ and $\left(\mathrm{L}-\mathrm{Ala}_{i+2}\right) \mathrm{NH} \cdots$ OC ( $\left.\mathrm{L}-\mathrm{Ala}_{i}\right)$.

The third low-energy conformation is a lefthanded $\beta^{6.2}$-helix whose backbone conformation is $\mathrm{CE}^{*}\left(-97^{\circ}, 76^{\circ}, 159^{\circ},-168^{\circ}\right)$ with $\Delta E_{\mathrm{res}}=0.19 \mathrm{kcal} \mathrm{mol}^{-1}$ (Figure 2b) and its enantiomer (right-handed $\beta^{6.2}$-helix) is the 4 th low-energy conformation, i.e., $\mathrm{EC}^{*}\left(-159^{\circ}\right.$, $169^{\circ}, 96^{\circ},-76^{\circ}$ ), with $\Delta E_{\text {res }}=0.21 \mathrm{kcal} \mathrm{mol}^{-1}$. Above the EC* backbone conformation of the L-Ala-D-Ala sequence in the left-handed $\beta^{6.2}$. helix is found the 7th low-energy conformation ( $\Delta E=0.96 \mathrm{kcal} \mathrm{mol}^{-1}$ ) of Ac-L-Ala-D-AlaNHMe. ${ }^{27}$ Moreover, several stable $\beta^{6}$-helices were also found along the low-energy trough stretched in $\mathrm{CE}^{*}, \mathrm{DE}^{*}, \mathrm{EC}^{*}$, and $\mathrm{ED}^{*}$ regions of $\left(\phi_{\text {L-Ala }}, \psi_{\text {L-Ala }}, \phi_{\text {D-Ala }}, \psi_{\text {D-Ala }}\right)$-space within $\Delta E_{\mathrm{res}}<0.5 \mathrm{kcal} \mathrm{mol}^{-1}$ (see Figure 1c). Distributions of $-\mathrm{NH}$ and $-\mathrm{CO}$ groups along the helical axis in these helices are changed with slight change of backbone dihedral angles.

Left- and right-handed $\alpha$-helices were found at $\Delta E_{\mathrm{res}}=0.23$ and $0.24 \mathrm{kcal} \mathrm{mol}^{-1}$ as the 5 and 6 th low-energy conformations, respectively.

In relatively high-energy regions $(0.5 \leqq$ $\Delta E_{\mathrm{res}} \leqq 2.5 \mathrm{kcal} \mathrm{mol}^{-1}$ ), seven groups of $\beta$ helices were found (Table I). The left-handed $\beta^{5.5}$-helix with $\Delta E_{\text {res }}=0.66 \mathrm{kcal} \mathrm{mol}^{-1}$ has smaller inside-radii of helix-cylinder than that of left-handed $\beta^{6.2}$-helix with $\Delta E_{\text {res }}=0.19$ $\mathrm{kcal} \mathrm{mol}^{-1}$. Both these $\beta$-helices exist in the $\mathrm{CE}^{*}$ region and difference between their backbone dihedral angles, $\left(\Delta \phi_{\mathrm{L}-\mathrm{Ala}}, \Delta \psi_{\mathrm{L}-\mathrm{Ala}}\right.$, $\left.\Delta \phi_{\mathrm{D}-\mathrm{Ala}}, \Delta \psi_{\mathrm{D}-\mathrm{Ala}}\right)$, is small, i.e., $\left(9^{\circ},-11^{\circ},-12^{\circ}\right.$, $5^{\circ}$ ). These results indicate that inside-radii of helix-cylinder, which works as ion channel in gramicidin $\mathrm{A}$, is sensitively changed by the small change of backbone dihedral angles in the $\mathrm{CE}^{*}$ region. In the energy range of $1.0<\Delta E_{\text {res }}<1.3 \mathrm{kcal} \mathrm{mol}^{-1}, \beta^{8.2}-, \beta^{10.2}$ and $\beta^{12.2}$-helices were found, and all of them situated in the $\mathrm{EE}^{*}$ region. Moreover, three helices composed of $\beta$-bend structure were found in the energy range of $1.8<$ $\Delta E_{\mathrm{res}}<2.1 \mathrm{kcal} \mathrm{mol}^{-1}$. The left-handed $\beta^{5.0}$. helix with $\Delta E_{\text {res }}=1.87 \mathrm{kcal} \mathrm{mol}^{-1}$ has typeII' bend at D-Ala-L-Ala sequence and the $-\mathrm{NH}$ and $-\mathrm{CO}$ groups of this helix are situated inside and outside the helix, respectively. Two helices with $\Delta E_{\mathrm{res}}=1.93$ and $2.07 \mathrm{kcal} \mathrm{mol}^{-1}$ have type-II bend at L-Ala-D-Ala sequences. The former helix corresponds to the LD-ribbon structure proposed by Ramachandran et al. ${ }^{22}$ and the latter one is right-handed $\beta^{4.3}$-helix which can be considered a special case of $\gamma$ helix found by conformational analysis on poly(Val-Pro-Gly-Gly). ${ }^{36}$

Energy difference between enantiomers for each helix is small, i.e., 0.07, 0.02 and $0.01 \mathrm{kcal} \mathrm{mol}^{-1}$ per residue for $\beta^{4.6}, \beta^{6.2}$ and $\alpha$ helices, respectively. These results indicate that the number of residues (24) used in this work is enough for the analysis of relative stabilities of helical conformations.

\section{DISCUSSIONS}

Comparison with the Experimental Results on Synthetic L-D Copolypeptides

By using NMR and IR spectroscopies ${ }^{16,19}$ and X-ray single-crystal diffraction analysis, ${ }^{18,20}$ the conformational characteristics of Boc-(L-Val-D-Val) ${ }_{n}$-OME ( $n=4,6$ and 8$)$ were investigated. Lorenzi et al. reported that Boc(L-Val-D-Val) ${ }_{4}$-OMe forms a double-stranded antiparallel $\beta^{5.6}$-helix as one of the stable conformations in cyclohexane and chloroform solutions. ${ }^{19}$ Moreover, they showed that Boc(L-Val-D-Val) 6 -OMe forms right- and lefthanded $\beta^{4.4}$-helices and the double-stranded left-handed antiparallel $\beta^{5.6}$-helix in chloroform solutions, ${ }^{16}$ but that only right- and left-handed $\beta^{4.4}$-helices are found for Boc-(LVal-D-Val) ${ }_{8}$-OMe in chloroform solutions. ${ }^{16}$ 
Benedetti et al. ${ }^{18,20}$ showed that Boc-(L-Val-D$\mathrm{Val})_{4}$-OMe forms a double-stranded lefthanded antiparallel $\beta^{5.6}$-helix in the crystal state. The crystal structure of Boc-(L-Phe-D$\mathrm{Phe}_{4}$-OMe was determined by X-ray diffraction analysis ${ }^{21}$ and also a double-stranded right-handed antiparallel $\beta^{5.6}$-helix, was proposed.

Conformations of poly( $\gamma$-benzyl-L-glutamyl- $\gamma$-bnzyl-D-glutamate) (abbreviated as poly(BLG-BDG)) in solution and solid state were analyzed by using NMR, CD and IR spectroscopies $^{11,12,14,15,17}$ and X-ray and electron diffraction measurements ${ }^{10,13}$ by Heitz et al. They reported that poly(BLG-BDG) takes a $\beta^{4.4}$-helix in dioxane, chloroform and DMF at high temperature $\mathrm{e}^{11,12,14,15}$, and that the $\beta^{4.4}$. helix is one of the stable conformations in the crystal state ${ }^{10,13}$, but that double-stranded $\beta$ helices and the $\alpha$-helix are also stable conformations in dioxane, chloroform and dichloromethane at low temperature or at high concentrations. ${ }^{11,12,14,15,17}$

Above experimental results correspond to our theoretical results. That is, the righthanded $\beta^{4.6}$-helix is the lowest-energy conformation and left-handed $\beta^{4.6}$-helix is the second low-energy one $\left(\Delta E_{\mathrm{res}}=0.07 \mathrm{kcal} \mathrm{mol}^{-1}\right)$. Good agreement between experimental and theoretical results indicates that peptides composed of alternating $\mathrm{D}$ - and L-amino acid sequences essentially have the tendency to form the $\beta^{4.6}$-helix within intra-molecular interactions. However, the energy differences between $\beta^{4.6}$-helix and othe conformations are not so significant, i.e., $\Delta E_{\mathrm{res}}=0.19,0.21,0.23$, and $0.24 \mathrm{kcal} \mathrm{mol}^{-1}$ for left- and right-handed $\beta^{6.2}$-helices, and left- and right-handed $\alpha$-helices, respectively. These results show that the above helical conformations besides $\beta^{4.6}$-helix can also exist depending on external conditions.

\section{Comparison with Experimental Results on Gramicidin A}

Urry et al. proposed the head-to-head $\beta^{6}$ - helical dimer at the amino termini ${ }^{4}$ as a model of gramicidin A in phospholipid bilayer membranes, and this conformation was detected by experimental works such as CD and NMR measurements ${ }^{4,6}$ and conductance study ${ }^{5}$. As shown in Table I, $\beta^{6.2}$-helical conformations are stable conformations of Ac-(L-Ala-DAla $)_{12}$-NHMe, and energy difference from the lowest-energy conformation $\left(\beta^{4.6}\right.$-helix $)$ is not so large $\left(\Delta E_{\mathrm{res}}=0.19\right.$ and $0.21 \mathrm{kcal} \mathrm{mol}^{-1}$ for left- and right-handed $\beta^{6.2}$-helices, respectively). These results indicate that the intramolecular interactions of Ac-(L-Ala-D-Ala) ${ }_{12}-$ $\mathrm{NHMe}$ can express basic conformational preference on the backbone structure of gramicidin A molecule, and that the native structure of gramicidin $\mathrm{A}$ is stabilized by the additional precise side-chain/side-chain and intermolecular interactions with lipid molecules in membranes.

As already mentioned in the RESULTS section, the theoretical results show that several $\beta^{6}$-helices can exist as stable conformations, and also that they are distributed in the neighboring region in $\left(\phi_{\mathrm{L}-\mathrm{Ala}}, \psi_{\mathrm{L}-\mathrm{Ala}}, \phi_{\mathrm{D}-\mathrm{Ala}}\right.$, $\left.\psi_{\text {D-Ala }}\right)$ space with close energy values, i.e., $0.27<\Delta E_{\text {res }}<0.40$. However, arrangements of carbonyl and amino groups along the inner helix-wall were different among these $\beta^{6}$-helices. These results suggest that the mechanism of cation-selection and transport through the membrane can be explained by conformational varieties of $\beta^{6}$-helices.

\section{Comparison with the Previous Theoretical Results}

Stable conformations of (L-Ala-D-Ala) were theoretically analyzed by RamachandranChandrasekaran, ${ }^{22}$ Hesselink-Scheraga ${ }^{23}$ and Colonna-Cesari et al. ${ }^{25}$ by the procedure of conformational energy calculations. Several helical structures $\left(\alpha\right.$-helix, $\beta^{4}-, \beta^{6}$-, and $\beta^{8}$-helices, and LD-ribbon structures) were proposed as stable conformations of (L-AlaD-Ala $)_{n}$. Hesselink-Scheraga ${ }^{23}$ and Colonna-Cesari et al. $^{25}$ used the optimization- 
Table II. Relative conformational energies ${ }^{a}$ of helical conformations

\begin{tabular}{lcccc}
\hline Helix & $\begin{array}{c}\text { Ramachandran- } \\
\text { Chandrasekaran }^{\mathrm{b}}\end{array}$ & $\begin{array}{c}\text { Hesselink- } \\
\text { Scheraga }^{\mathrm{c}}\end{array}$ & $\begin{array}{c}\text { Colonna-Cesari } \\
\text { et al. }^{\text {d }}\end{array}$ & This work \\
\hline$\alpha$-helix (R) & - & 0.09 & 0.00 & 0.24 \\
$\alpha$-helix (L) & - & 0.00 & - & 0.23 \\
$\beta^{4}$-helix (R) & 0.0 & - & $0.61\left(\beta^{4.4}\right)$ & $0.00\left(\beta^{4.6}\right)$ \\
$\beta^{4}$-helix (L) & - & - & - & $0.07\left(\beta^{4.6}\right)$ \\
$\beta^{6}$-helix (R) & 0.75 & 2.52 & - & $0.21\left(\beta^{6.2}\right)$ \\
$\beta^{6}$-helix (L) & - & - & - & $0.19\left(\beta^{6.2}\right)$ \\
$\beta^{8}$-helix (R) & 2.6 & - & $-1.13\left(\beta^{8.2}\right)$ \\
$\beta^{8}$-helix (L) & - & & - & $1.09\left(\beta^{8.2}\right)$ \\
\hline
\end{tabular}

a Relative conformational energy $\left(\mathrm{kcal} \mathrm{mol}^{-1}\right)$ per residue.

b From ref 22 .

c From ref 23.

d From ref 25.

e Helix sense is abbreviated as $\mathrm{R}$ or L for right- and left-hand, respectively.

procedure and showed that the $\alpha$-helix is the molst stable conformation. The former results show that $\beta^{6}$-helical conformations is less stable than $\alpha$-helical ones with $\Delta E_{\text {res }}=$ $2.52 \mathrm{kcal} \mathrm{mol}^{-1}$, and the latter ones also show that $\beta^{4.4}$ - and $\beta^{6.3}$-helical conformations are less stable than $\alpha$-helical one with $\Delta E_{\mathrm{res}}=0.61$ and $0.63 \mathrm{kcal} \mathrm{mol}^{-1}$, respectively. Ramachandran-Chandrasekaran ${ }^{22}$ showed that the $\beta^{4}$-helix is the most stable conformation among $\beta^{4}-, \beta^{6}$-, and $\beta^{8}$-helices and the LD-ribbon structure. However, conformational energy of the $\alpha$-helical structure was not presented in their results, and the conformational energies were not optimized in their calculations. Calculated results of the above three works are summerized in Table II with our calculated results.

Our calculated results show that $\beta^{4.6}$-helix is the most stable conformation for Ac-(L-Ala-DAla) ${ }_{12}$-NHMe, and that left-handed $\beta^{6.2}$-helix and right-handed $\alpha$-helix are also stable with small energy differences, i.e., $\Delta E_{\mathrm{res}}=0.19$ and $0.24 \mathrm{kcal} \mathrm{mol}^{-1}$, respectively. Moreover, our results also correspond to the experimental results that the $\beta^{4}$-helix is a stable conformation for poly(L-Val-D-Val) and poly(BLGBDG) and it can be transformed to the $\alpha$-helix with change in temperature.
The universality of the method used in searching for stable helical conformations is very important for analyzing the relative stabilities of helical conformaion. Previous three works ${ }^{22,23,25}$ are based on the assumption that helical structures are essentially stabilized by hydrogen bonds. Either the particular helical conformations speculated by the patterns of hydrogen-bond formation or those corresponding to the estimated helical parameters such as $n$ (number of residues per turn) and $h$ (the rise per residue) were analyzed. In this work, we used no assumptions of previous theoretical works on helical structures, but only the hypothesis for the hierarchy on interactions in polypeptide molecules mentioned in the THEORETICAL section. That is, we selected all starting conformations found as stable ones for both L-Ala and D-Ala residues with intra-residue interactions, and searched for the helical conformations by stepwise expansion of the interaction-level from shortrange to long-range. Good agreement between theoretical and experimental results indicates the validity of the hypothesis used in this work.

\section{REFERENCES}

1. V. T. Ivanov and S. V. Sychev, Proceedings, 
Workshop Conference, Hoechst, Vol. 11, 1981, p 107.

2. P. Läuger, Angew. Chem. Int. Ed. Engl., 24, 905 (1985).

3. P. C. Jordan, J. Phys. Chem., 91, 6582 (1987).

4. D. W. Urry, M. C. Goodall, J. D. Glickson, and D. F. Mayers, Proc. Natl. Acad. Sci. U.S.A., 68, 1907 (1971).

5. E. Bamberg, H. J. Apell, and H. Alpes, Proc. Natl. Acad. Sci. U.S.A., 74, 2402 (1977).

6. S. Weinstein, B. A. Wallace, E. R. Blout, J. S. Morrow, and W. R. Veatch, Proc. Natl. Acad. Sci. U.S.A., 76, 4230 (1979).

7. W. R. Veatch, E. T. Fossel, and E. R. Blout, Biochemistry, 13, 5249 (1974).

8. B. W. Wallace, Biophys. J., 49, 295 (1986).

9. D. A. Langs, Biopolymers, 28, 259 (1989).

10. F. Heitz, B. Lotz, and G. Spach, J. Mol. Biol., 92, 1 (1975).

11. F. Heitz and G. Spach, Macromolecules, 8, 740 (1975).

12. F. Heitz, P. D. Cary, and C. Crane-Robinson, Macromolecules, 8, 745, (1975).

13. B. Lotz, F. Colonna-Cesari, F. Heitz, and G. Spach, J. Mol. Biol., 106, 915 (1976).

14. F. Heitz and G. Spach, Macromolecules, 10, 520 (1977).

15. F. Heitz, P. D. Cary, and C. Crane-Robinson, Macromolecules, 10, 526 (1977).

16. G. P. Lorenzi, H. Jäckle, L. Tomasic, and C. Pedone, Biopolymers, 22, 157 (1983).

17. F. Heitz, Macromolecules, 10, 1289 (1977).

18. E. Benedetti, B. Di Blasio, C. Pedone, G. P. Lorenzi, L. Tomasic, and V. Gramlich, Nature, 282, 630 (1979).
19. G. P. Lorenzi, H. Jackle, L. Tomasic, V. Rizzo, and C. Pedone, J. Am. Chem. Soc., 104, 1728 (1982).

20. B. Di Blasio, E. Benedetti, V. Pavone, C. Pedone, O. Spiniello, and G. P. Lorenzi, Biopolymers, 28, 193 (1989).

21. B. Di Blasio, E. Benedetti, V. Pavone, C. Pedone, C. Gerber, and G. P. Lorenzi, Biopolymers, 28, 203 (1989).

22. G. N. Ramachandran and R. Chandrasekaran, Indian J. Biochem. Biophys., 9, 1 (1972).

23. F. T. Hesselink and H. A. Scheraga, Macromolecules, 5, 455 (1972).

24. P. De Santis, S. Morosetti and R. Rizzo, Macromolecules, 7, 52 (1974).

25. F. Colonna-Cesari, S. Premilat, F. Heitz, G. Spach, and B. Lotz, Macromolecules, 10, 1284 (1977).

26. C. M. Venkatachalam and D. W. Urry, J. Comput. Chem., 4, 461 (1983).

27. M. Oka and A. Nakajima, Polym. J., 16, 553 (1984).

28. G. Nemethy and H. A. Scheraga, Qurt. Rev. Biophys., 10, 239 (1977).

29. F. A. Momany, R. F. McGuire, A. W. Burgess and H. A. Scheraga, J. Phys. Chem., 79, 2361 (1975).

30. M. J. D. Powell, Computer J., 7, 155 (1964).

31. S. S. Zimmerman and H. A. Scheraga, Biopolymers, 16, 811 (1977).

32. S. S. Zimmerman, M. S. Pottle, G. Nemethy, and H. A. Scheraga, Macromolecules, 10, 1 (1977).

33. R. A. Scott and H. A. Scheraga, J. Chem. Phys., 45, 2091 (1966).

34. T. Ooi, R. A. Scott, G. Vanderkooi, and H. A. Scheraga, J. Chem. Phys., 46, 4410 (1967).

35. M. Sisido, private communications.

36. M. Oka, Y. Baba, A. Kagemoto, and A. Nakajima, submitted for publication. 